\section{Multimorbidität häufig bei rheumatoider Arthritis}

\author{
England BR et al. Burden and trajectory of \\ multimorbidity in rheumatoid arthritis: a \\ matched cohort study from 2006 to 2015. \\ Annals of the rheumatic diseases 2021; 80: \\ 286-292. doi:10.1136/annrheum- \\ dis-2020-218282
}

Im Jahr 2014 litten über $\mathbf{4 0} \%$ aller Erwachsenen in den USA an mehreren chronischen Erkrankungen. Einzelne Komorbiditäten verkomplizierten bekanntlich die rheumatoide Arthritis (RA). Der Zeitpunkt und die Häufigkeit des Auftretens von Multimorbidität bei RA war bislang aber unbekannt. Eine US-amerikanische Studie zeigt nun, dass die Belastung durch Multimorbidität und die Häufigkeit der Entstehung chronischer Erkrankungen bei RA größer ist.

Dies konnte in einer gematchten, retrospektive Kohortenstudie festgestellt werden, in die Daten aus einer großen, kommerziellen US-Versicherungsdatenbank (MarketScan) von 2006 bis 2015 eingeflossen sind. Ziel war ein Vergleich des Auftretens und des Verlaufs von Multimorbidität bei Personen mit und ohne RA. Unter Verwendung validierter Algorithmen wurden Patienten mit RA mit Patienten ohne RA anhand des Geschlechtes und des Alters gematcht. Die Forscherinnen und Forscher wählten diagnostische Codes für 44 vordefinierte chronische Erkrankungen aus, um das Vorhandensein ( $\geq 2$ Erkrankungen) und die Belastung (Anzahl) von Multimorbidität zu bestimmen. Es folgten Querschnittsvergleiche unter Verwendung der gesamten RA-Kohorte und bedingter logistischer und negativer binomialer Regressionsmodelle. Verläufe der Multimorbidität wurden innerhalb der inzidenten RA-Subkohorte unter Verwendung verallgemeinerter Schätzungsgleichungen bewertet.

Von mehr als 147 Mio. Personen in der Datenbank wurden 138891 ausgewählt, welche die Zulassungskriterien und den RA-Algorithmus erfüllten, darunter 30562 mit inzidenter RA. Nach dem Matching (1:1) waren in der Gesamtkohorte $n=277782$ und in der Inzidenz-Subkohorte $\mathrm{n}=61124$ Personen. Die eingeschlossenen Teilneh- mer waren überwiegend weiblich (76,5\% bzw. 74,1\%) mit einem Durchschnittsalter von 55,6 Jahren bzw. 54,5 Jahren.

\section{Ergebnisse}

- Die querschnittliche Prävalenz (OR 2,29; $95 \%-K I$ 2,25-2,34) und Belastung (Verhältnis von Erkrankungen 1,68; $95 \%-K I$ 1,66-1,70) der Multimorbidität waren bei RA signifikant höher als ohne RA in der gesamten Kohorte.

- Die am meisten überrepräsentierten chronischen Erkrankungen bei RA waren interstitielle Lungenerkrankung (OR 12,62; $95 \%-K I$ 10,54-15,11), Fibromyalgie (OR 5,86; $95 \%-K I$ 5,50-6,25), Osteoarthritis (OR 5,16; $95 \%-K I 4,98-5,35)$ und Osteoporose (OR 4,54; $95 \%-K I$ 4,19-4,92).

- Innerhalb der inzidenten RA-Kohorte hatten die Patienten mit RA mehr chronische Erkrankungen als Nicht-RAPatienten ( $\beta 1,13,95 \%-K I 1.10-1.17)$. Die Rate des Auftretens von chronischen Erkrankungen war signifikant höher bei RA im Vergleich zu Nicht-RA (RA $\times$ Nachbeobachtungsjahr $\beta 0,21$; $95 \%-K I$ $0,20-0,21 ; p<0.001)$.

- Andere Faktoren, die mit einer größeren Multimorbidität verbunden waren, waren weibliches Geschlecht, höheres Alter und eine längere Dauer der Nachbeobachtung.

- Die Ergebnisse waren auch dann ähnlich, wenn die Prä-RA Periode eingeschlossen wurde und wenn mehrere Sensitivitätsanalysen durchgeführt wurden.

\section{FAZIT}

Laut den Autoren ist Multimorbidität bei RA hochprävalent. Sie schreite bei Patienten mit RA während und unmittelbar nach Ausbruch der RA schneller voran als bei Patienten ohne RA. Die Multimorbidität solle daher frühzeitig im Krankheitsverlauf erkannt und gezielt behandelt werden.

Einschränkung der Studie sei beispielsweise die mangelnde Adjustierung für Gesundheitsverhalten und soziodemografische Daten, was zu Konfundierung geführt haben könnte.

Annkatrin Wagner, Stuttgart 